\section{Bone Mass and Density in Youth With Type 2 Diabetes, Obesity, and Healthy Weight}

Diabetes Care 2020;43:2544-2552 | https://doi.org/10.2337/dc19-2164
Joseph M. Kindler, ${ }^{1}$ Andrea Kelly, ${ }^{2,3}$ Philip R. Khoury, ${ }^{4}$ Lorraine E. Levitt Katz, ${ }^{2,3}$ Elaine M. Urbina, ${ }^{4,5}$ and Babette S. Zemel ${ }^{1,3}$

\section{OBJECTIVE}

Youth-onset type 2 diabetes is an aggressive condition with increasing incidence. Adults with type 2 diabetes have increased fracture risk despite normal areal bone mineral density (aBMD), but the influence of diabetes on the growing skeleton is unknown. We compared bone health in youth with type 2 diabetes to control patients with obesity or healthy weight.

\section{RESEARCH DESIGN AND METHODS}

Cross-sectional study of youth (56\% African American, 67\% female) ages 10-23 years with type 2 diabetes ( $n=180$ ), obesity (BMI >95th; $n=226$ ), or healthy weight (BMI <85th; $n=238$ ). Whole-body (less head) aBMD and lean mass as well as abdominal visceral fat were assessed via DXA. Lean BMI (LBMI) and aBMD SD scores ( $z$ scores) were computed using published reference data.

\section{RESULTS}

We observed age-dependent differences in aBMD and LBMI $z$ scores between the healthy weight, obese, and type 2 diabetes groups. In children, aBMD and LBMI $z$ scores were greater in the type 2 diabetes group versus the obese group, but in adolescents and young adults, aBMD and LBMI $z$ scores were lower in the type 2 diabetes group versus the obese group (age interactions $P<0.05$ ). In the type 2 diabetes group and the obese group, aBMD was about 0.5 SDs lower for a given LBMI $z$ score compared with healthy weight control patients $(P<0.05)$. Further, aBMD was lower in those with greater visceral fat $(\beta=-0.121, P=0.047)$.

\section{CONCLUSIONS}

These results suggest that type 2 diabetes may be detrimental to bone density around the age of peak bone mass. Given the increased fracture risk in adults with type 2 diabetes, there is a pressing need for longitudinal studies aimed at understanding the influence of diabetes on the growing skeleton.

Type 2 diabetes has historically been considered an adult-onset condition, but it was increasingly recognized as occurring in children and adolescents in the 1990s (1). Epidemiological data from the SEARCH for Diabetes in Youth (SEARCH) study indicated that type 2 diabetes incidence in U.S. youth has increased about $5 \%$ per year since the early 2000 s (2). Youth-onset type 2 diabetes is an aggressive condition characterized by insulin resistance and reduced $\beta$-cell function, and it is associated with accelerated onset of complications including hypertension, hyperlipidemia, vascular dysfunction, systemic inflammation, and renal hyperfiltration (3-5). Adults with type 2 diabetes have increased fracture risk despite having normal or increased areal bone mineral
${ }^{1}$ Division of Gastroenterology, Hepatology and Nutrition, Children's Hospital of Philadelphia, Philadelphia, PA

${ }^{2}$ Division of Endocrinology and Diabetes, Children's Hospital of Philadelphia, Philadelphia, PA ${ }^{3}$ Department of Pediatrics, University of Pennsylvania Perelman School of Medicine, Philadelphia, PA

${ }^{4}$ The Heart Institute, Cincinnati Children's Hospital Medical Center, Cincinnati, $\mathrm{OH}$

${ }^{5}$ Department of Pediatrics, University of Cincinnati, Cincinnati, $\mathrm{OH}$

Corresponding author: BabetteS. Zemel, zemel@ email.chop.edu

Received 29 October 2019 and accepted 9 July 2020

This article contains supplementary material online at https://doi.org/10.2337/figshare.12665486

E.M.U. and B.S.Z. contributed equally to this study.

(C) 2020 by the American Diabetes Association. Readers may use this article as long as the work is properly cited, the use is educational and not for profit, and the work is not altered. More information is available at https://www.diabetesjournals .org/content/license. 
density (aBMD) $(6,7)$. Similarly, children with obesity have normal or increased aBMD, yet epidemiological studies report that obese youth are overrepresented in fracture cases (8). Several studies have implicated obesity-related metabolic factors, such as insulin resistance (9), impaired glucose tolerance (10), the metabolic syndrome (11), and prediabetes (12) as potential detriments to childhood bone health. Pathogenic fat depots, notably abdominal visceral fat, have also been hypothesized to contribute to these findings $(11,13)$. Despite having greater body size and body weight, mice with early onset type 2 diabetes display lower whole-body BMD compared with nonobese controls without diabetes (14). However, clinical evidence involving bone mass and density in youth with type 2 diabetes is lacking.

Skeletal muscle is a strong determinant of bone accrual during childhood, yet the role of muscle in bone development might be altered in individuals with excess adiposity and insulin resistance (15). Additionally, mouse models suggest that the influence of type 2 diabetes on bone acquisition is dependent on age, with the greatest effect being evident at later stages of development (14). Similar relationships were reported in adults with type 2 diabetes, wherein individuals with a longer time since diagnosis exhibited the greatest bone deficits (16). These observations highlight the importance of investigating the relationships between type 2 diabetes and bone outcomes across critical periods of bone accrual and peak bone mass attainment, which is typically achieved around the age of 20 years (17).

We performed a secondary analysis of previously acquired data from youth ages 10-23 years with type 2 diabetes, obesity, and healthy weight. Our primary objective was to compare bone mass and density in youth with type 2 diabetes to control patients with obesity and healthy weight. Additional analyses were performed to compare bone and lean mass $z$ score differences across groups relative to age and lean mass $z$ scores and to investigate relationships between abdominal fat depots and bone outcomes.

\section{RESEARCH DESIGN AND METHODS}

This was a secondary analysis of previously acquired data from African American and non-African American males and females, ages $10-23$ years, with type 2 diabetes $(n=180)$, obesity ( $n=$ $226)$, and healthy weight $(n=238)(4,18)$. Participants were primarily recruited from the Diabetes Clinic at Cincinnati Children's Hospital Medical Center, as well as from local physician offices, college campuses, and health fairs in Cincinnati, $\mathrm{OH}$. Females who were pregnant were excluded from participating in this study. All data collection was performed at Cincinnati Children's Hospital Medical Center.

Diagnosis of type 2 diabetes was based on the criteria of the American Diabetes Association (19). About $8 \%(n=15)$ of the patients in the type 2 diabetes group were diagnosed after age 18 years. For subjects $>20$ years of age, all anthropometric and bone $z$ scores were calculated using an age of 20 years. Healthy weight was defined as BMI-for-age percentile between the 5th and 85th, and obese was defined as BMI-for-age percentile $>95$ th. Subjects with type 2 diabetes were negative for $G A D$, islet cell autoantigen 512, and insulin autoantibodies. Individuals in the obese group were determined to not have impaired glucose tolerance or type 2 diabetes based on an oral glucose tolerance test. Subjects in the healthy weight and obese groups with glycosylated hemoglobin $\mathrm{A}_{1 \mathrm{c}}\left(\mathrm{HbA}_{1 \mathrm{c}}\right)$ $>6.5 \%$ or fasting glucose $>126 \mathrm{mg} / \mathrm{dL}$ were excluded from all analyses. Use of metformin or insulin was queried via selfreport.

Written informed consent or assent was obtained from each subject or legal guardian. The Institutional Review Board for Human Subjects at Cincinnati Children's Hospital Medical Center approved all study protocols and procedures.

\section{Anthropometric Measurements and Maturation}

Anthropometric measurements were performed while subjects wore light indoor clothing. Height was measured using a wall-mounted stadiometer (Veeder-Rood, Elizabethtown, NC), and weight was measured using an electronic scale (Health-OMeter). SD scores ( $z$ scores) for height, weight, and BMI were calculated using Centers for Disease Control and Prevention reference data (20). A self-assessment questionnaire that included pictograms and written descriptions was used to assess breast (females) or pubic hair (males) development. Subjects were subsequently categorized into stages of maturation according to the method of Tanner (21).

\section{Plasma Biochemistries}

Blood samples were collected in the morning following a 10-h overnight fast. Plasma glucose and insulin, serum $25(\mathrm{OH}) \mathrm{D}$, and $\mathrm{HbA}_{1 \mathrm{c}}$ were assessed $(4,18,22)$.

\section{DXA}

DXA scans were obtained using a Hologic QDR 4500A scanner (Hologic, Inc., Bedford, MA) and were analyzed using Apex software (version 5.5.3). Wholebody (less head) bone mineral content (BMC) and aBMD, lean mass, and abdominal visceral $\left(\mathrm{cm}^{2}\right)$ and subcutaneous $\left(\mathrm{cm}^{2}\right)$ fat area were assessed. Lean BMI (LBMI) was calculated as lean mass (kg)/ height $(\mathrm{m})^{2}$. The $z$ scores for BMC, aBMD (23), and LBMI (24) were calculated using previously published reference data. Since whole-body BMC and aBMD are confounded by stature, bone $z$ scores were subsequently adjusted for height $z$ score (25).

\section{Dietary Intake}

Dietary intake was assessed using 3-day records, which were analyzed using the Nutrition Data System for Research. Variables of interest included 3-day averages for total energy (kcal/day) and calcium (mg/day).

\section{Statistical Analysis}

All variables were evaluated for outliers and implausible data points. Betweengroup comparisons were performed using ANOVA or Kruskal-Wallis/Dunn tests with Bonferroni post hoc adjustment. Categorical variables were compared across groups using Pearson $\chi^{2}$ tests. Time since type 2 diabetes diagnosis (square root) and 25(OH)D (log) were transformed to follow an approximately normal distribution. The bivariate association between time since diagnosis and age was assessed using Pearson correlation.

Linear regression was used to assess differences in anthropometric, LBMI, and bone $z$ scores between type 2 diabetes, obese, and healthy weight groups relative to age. For these analyses, main effects for group (categorical variable) and age (continuous variable), and age by group interactions were included as model parameters. Similar analyses assessed group differences in bone $z$ scores 
relative to LBMI $z$ scores. For these analyses, main effects for group and LBMI $z$ score (continuous variable), and LBMI $z$ score by group interactions were included as model parameters. The healthy weight group was used as the reference for each of these analyses, and the "lincom" command in STATA was used to compare coefficients between the patients in the obese and type 2 diabetes groups. To assist in interpreting these results, regression models were subsequently used to calculate predicted $z$ scores for a given age using the minimum and maximum age within each group, which was 10.1 and 24.0, respectively, for the healthy weight group; 10.4 and 23.9, respectively, for the obese group; and 10.2 and 23.8 , respectively, for the type 2 diabetes group. Similarly, predicted bone $z$ scores for a given LBMI $z$ score were calculated using LBMI values of -0.15 and 1.05 , which represent the range within which LBMI $z$ scores overlapped for the three groups. Several sensitivity analyses were performed: 1) sex, ancestry, maturation, and 25(OH)D were included as covariates in all regression analyses; 2) three-way interactions between group, age, and sex were investigated with respect to bone/LBMI $z$ scores; and 3 ) individuals in the type 2 diabetes group with a BMI less than the 95th percentile were excluded from all regression analyses. Unless otherwise indicated, results of the sensitivity analyses were consistent with the models presented. The three-way interaction between group, age, and sex were not statistically significant and are not presented below.

Relationships between abdominal visceral and subcutaneous fat and bone outcomes were assessed using multiple linear regression. These adiposity measures covaried strongly with weight status, so the healthy weight group was analyzed separately from the obese and type 2 diabetes groups to permit detection of potential effects of different adipose depots on bone outcomes. These models included visceral and subcutaneous fat, ancestry, sex, and age as model parameters. Additional analyses included 25(OH)D. For regression analyses, collinearity and normality of regression residuals were inspected. All statistical analyses were performed using STATA (version 15.1) and RStudio (version 1.1.463). $P$ values $<0.05$ were considered statistically significant.

\section{RESULTS}

\section{Sample Description}

Sample characteristics are displayed in Table 1. Across the three groups, there were significant differences in ancestry, maturation stage, weight, BMI, BMC, aBMD, LBMI, visceral fat, subcutaneous fat, $\mathrm{HbA}_{1 \mathrm{c}}$, insulin, and glucose (all $P<$ $0.05)$. BMC and aBMD $z$ scores were greater in the type 2 diabetes and obese groups versus the healthy weight group (all $P<0.001$ ), but they were similar between the type 2 diabetes and obese groups. Weight, BMI, and LBMI $z$ scores, visceral and subcutaneous fat, $\mathrm{HbA}_{1 \mathrm{c}}$, insulin, and glucose were greater in the type 2 diabetes and obese groups versus the healthy weight group (all $P<0.05$ ). Visceral fat, $\mathrm{HbA}_{1 \mathrm{c}}$, and glucose were greater in the type 2 diabetes group versus the obese group (both $P<0.05$ ). Youth with healthy weight reported greater calcium and energy intake and had greater 25(OH)D compared with the youth in the obese and type 2 diabetes groups (all $P<0.001$ ). In the type 2 diabetes group, about $62 \%$ of patients reported using metformin and about $51 \%$ reported using insulin.

In the type 2 diabetes group, age at diagnosis ranged from 6.4 to 20.5 and time since diagnosis ranged from 0.1 to 12.3 years. The amount of time since diagnosis correlated positively with age $(r=0.62, P<0.001)$

\section{Differences in Height and LBMI $z$ Scores According to Age}

Linear regression analyses investigating age-related trends in height and LBMI z scores are presented in Supplementary Table 1, and visual representations of these relationships are displayed in Fig. $1 A$ and $B$, respectively. For height $z$ score, there was a significant age by group interaction $(P<0.05)$, meaning that at younger ages, the patients in the type 2 diabetes and obese groups were tallerfor-age compared with those in the healthy weight group, but differences in stature were not evident in adolescents and young adults. At age 10 years, predicted height $z$ scores in the healthy weight, obese, and type 2 diabetes groups were $0.19,0.75$, and 0.95, respectively, but at age 24 years, the values were $0.11,-0.23$, and -0.05 , respectively. Across the age range, the LBMI $z$ score was greater in the obese and type 2 diabetes groups versus the healthy weight group, but there was a significant age by group interaction $(P<0.05)$. The LBMI $z$ score was consistent across the age range in the obese and healthy weight groups, but the type 2 diabetes group showed an age-related decline in LBMI $z$ score. To illustrate, at age 10 years, predicted LBMI $z$ scores in the healthy weight, obese, and type 2 diabetes groups were -0.38 , 1.65 , and 2.10 , respectively, but at age 24 years, the scores were $-0.25,1.76$, and 1.10, respectively. Similar age-related differences between groups were observed for weight and BMI $z$ scores (Supplementary Table 1). When including vitamin $D$ in the model for height $z$ score, the age by group interactions for the obese $(P=0.080)$ and type 2 diabetes $(P=0.137)$ groups were attenuated.

\section{Differences in Bone $z$ Scores According to Age}

Linear regression analyses investigating age-related trends in BMC and aBMD $z$ scores are presented in Supplementary Table 2, and visual representations of these relationships are displayed in Fig. $1 C$ and $D$, respectively. Across the age range, bone $z$ scores were greater in the type 2 diabetes and obese groups versus the healthy weight group, but there was a significant age by group interaction $(P<$ $0.05)$. The bone $z$ scores were consistent across the age range in the obese and healthy weight groups, but the type 2 diabetes group showed an age-related decline in bone $z$ scores. To illustrate, at age 10 years, the predicted aBMD z scores for the healthy weight, obese, and type 2 diabetes groups were $0.36,0.78$, and 1.30 , respectively, but at age 24 years, the scores were $0.57,0.94$, and 0.57 , respectively. When including vitamin $D$ as an additional model parameter, the age by group interaction for BMC $z$ score remained significant $(P=0.006)$, but the aBMD $z$ score was not $(P=$ 0.157).

\section{Relationships Between LBMI and Bone $z$ Scores}

Linear regression analyses investigating relationships between LBMI and bone $z$ scores are presented in Supplementary Table 3, and they are visually represented in Fig. 2. The LBMI $z$ score was positively associated with BMC and aBMD $z$ scores (both $P<0.001$ ). However, for a given LBMI $z$ score, BMC and aBMD $z$ scores were lower in the type 2 diabetes and 
Table 1-Sample characteristics

\begin{tabular}{|c|c|c|c|c|}
\hline & Healthy weight $(n=238)$ & Obese $(n=226)$ & Type 2 diabetes $(n=180)$ & $P$ value $^{1}$ \\
\hline \multicolumn{5}{|l|}{ Demographics } \\
\hline Age (years) & $17.72 \pm 3.54$ & $18.07 \pm 3.20$ & $17.88 \pm 3.15$ & 0.535 \\
\hline Age at diagnosis (years) & & & $14.7(12.2-16.4)$ & \\
\hline Time since diagnosis (years) & & & $3.2(0.9-5.2)$ & \\
\hline Ancestry (\% African American) ${ }^{2}$ & 51.3 & 66.4 & 50.0 & 0.001 \\
\hline Sex $(\% \text { female })^{2}$ & 63.9 & 71.7 & 65.6 & 0.176 \\
\hline Maturation stage, $n(\%)^{2 *}$ & & & & $<0.001$ \\
\hline Stage 1 & $7(3.0)$ & $0(0.0)$ & $1(0.6)$ & \\
\hline Stage 2 & $8(3.4)$ & $1(0.5)$ & $3(1.7)$ & \\
\hline Stage 3 & 31 (13.1) & $21(9.4)$ & $16(9.0)$ & \\
\hline Stage 4 & $74(31.2)$ & $34(15.3)$ & $52(29.2)$ & \\
\hline Stage 5 & $117(49.4)$ & $167(74.9)$ & $106(59.6)$ & \\
\hline \multicolumn{5}{|l|}{ Anthropometrics } \\
\hline Height (z score) & $0.14 \pm 1.00$ & $0.20 \pm 1.11$ & $0.39 \pm 1.15$ & 0.068 \\
\hline Weight $(z \text { score })^{3}$ & $0.14(-0.30$ to 0.64$)$ & $2.33(2.01-2.64)^{\mathrm{a}}$ & $2.26(1.78-2.70)^{\mathrm{a}}$ & $<0.001$ \\
\hline BMI $(z \text { score })^{3}$ & $0.13(-0.44$ to 0.53$)$ & $2.47(1.81-3.18)^{\mathrm{a}}$ & $2.38(1.59-3.18)^{\mathrm{a}}$ & $<0.001$ \\
\hline \multicolumn{5}{|l|}{ DXA } \\
\hline BMC (z score $)^{3}$ & $0.22(-0.38$ to 0.61$)$ & $1.08(0.54-1.61)^{\mathrm{a}}$ & $1.01(0.45-1.55)^{\mathrm{a}}$ & $<0.001$ \\
\hline aBMD (z score) & $0.47 \pm 0.87$ & $0.87 \pm 0.93^{\mathrm{a}}$ & $0.88 \pm 1.02^{\mathrm{a}}$ & $<0.001$ \\
\hline LBMI ( $z$ score) & $-0.29(-0.79$ to 0.15$)$ & $1.70(1.32-2.09)^{\mathrm{a}}$ & $1.62(0.97-2.15)^{\mathrm{a}}$ & $<0.001$ \\
\hline Visceral fat $\left(\mathrm{cm}^{2}\right)^{3}$ & $32.6(23.4-42.5)$ & $97.1(77.6-122.8)^{\mathrm{a}}$ & $112.5(81.8-153.8)^{\mathrm{ab}}$ & $<0.001$ \\
\hline Subcutaneous fat $\left(\mathrm{cm}^{2}\right)^{3}$ & $159.7(84.7-213.4)$ & $565.7(454.9-681.4)^{a}$ & $533.3(427.5-653.7)^{\mathrm{a}}$ & $<0.001$ \\
\hline \multicolumn{5}{|l|}{ Dietary intake } \\
\hline Calcium (mg/day) & $928.0 \pm 455.1$ & $762.7 \pm 403.4^{\mathrm{a}}$ & $801.3 \pm 419.1^{\mathrm{a}}$ & $<0.001$ \\
\hline Calories (kcal/day) & $2,183 \pm 836$ & $2,029 \pm 747^{a}$ & $1,962 \pm 736^{\mathrm{a}}$ & $<0.001$ \\
\hline \multicolumn{5}{|l|}{ Biochemistries } \\
\hline $\mathrm{HbA}_{1 \mathrm{c}}(\%)^{3}$ & $5.3(5.1-5.5)$ & $5.5(5.2-5.7)^{\mathrm{a}}$ & $6.9(5.8-10.0)^{\mathrm{ab}}$ & $<0.001$ \\
\hline Insulin $(\mathrm{mlU} / \mathrm{L})^{3}$ & $10.0(8.3-13.2)$ & $18.2(13.7-26.8)^{\mathrm{a}}$ & $20.6(13.6-32.7)^{\mathrm{a}}$ & $<0.001$ \\
\hline Glucose $(\mathrm{mg} / \mathrm{dL})^{3}$ & $89.1(85.1-93.3)$ & $91.3(87.3-95.9)^{\mathrm{a}}$ & $116.8(92.8-199.6)^{\mathrm{ab}}$ & $<0.001$ \\
\hline $25(\mathrm{OH}) \mathrm{D}(\mathrm{ng} / \mathrm{mL})$ & $20.3(13.9-26.7)$ & $12.4(8.7-17.6)^{\mathrm{a}}$ & $13.0(8.5-18.7)^{a}$ & $<0.001$ \\
\hline \multicolumn{5}{|l|}{ Medication use (\% reported using) } \\
\hline Metformin & & & $62.2 \%$ & \\
\hline Insulin & & & $51.2 \%$ & \\
\hline
\end{tabular}

Values are mean \pm SD or median (interquartile range), unless otherwise noted. ${ }^{1} \mathrm{Tests}$ of significance performed using ANOVA with a Bonferroni post hoc adjustment. ${ }^{2}$ Test of significance performed using $\chi^{2}$ test. ${ }^{3}$ Test of significance performed using Kruskal-Wallis/Dunn test. ${ }^{2}$ Significantly different than healthy weight group $(P<0.05)$. ${ }^{b}$ Significantly different than obese group $(P<0.05) .{ }^{*} n=237$ for healthy weight, $n=223$ for obese, and $n=178$ for type 2 diabetes.

obese groups versus the healthy weight group (all $P<0.05$ ). For instance, at an LBMI $z$ score of -0.15 , predicted aBMD $z$ scores for the healthy weight, obese, and type 2 diabetes groups were $0.57,-0.11$, and 0.17 , respectively, and at a LBMI $z$ score of 1.05, aBMD $z$ scores were 1.33, 0.52 , and 0.68 , respectively. There were no differences in bone $z$ scores between the type 2 diabetes and obese groups relative to LBMI $z$ score.

\section{Relationships Between Abdominal Fat and Bone $z$ Scores}

Among the obese and type 2 diabetes groups, the multivariate regression models including age, ancestry, and sex showed that subcutaneous fat was positively associated with BMC $z$ scores but that visceral fat was negatively associated with BMC and aBMD $z$ scores (all $P<$ 0.05 ) (Table 2). In additional analyses that included vitamin $D$, serum $25(\mathrm{OH}) \mathrm{D}$ was positively associated with $z$ scores for BMC ( $\beta=0.131, P=0.045)$ and aBMD ( $\beta=0.146, P=0.032$ ), and subcutaneous fat was positively associated with the BMC $z$ score $(\beta=0.230, P=0.001)$. However, the inverse relationship between visceral fat and aBMD was attenuated $(P=$ 0.159 ). Visceral and subcutaneous fat were not significantly associated with bone $z$ scores in the healthy weight group alone.

\section{CONCLUSIONS}

This is the first study to report on bone health in youth with type 2 diabetes. We observed age-dependent differences in skeletal measures between individuals with type 2 diabetes and obesity that suggest an adverse influence of diabetes on aBMD during the critical window of peak bone mass attainment. Specifically, aBMD and LBMI $z$ scores were higher among children in the type 2 diabetes versus the obese group, but they were lower among adolescents and young adults in the type 2 diabetes group versus the obese group. In other words, with the transition to young adulthood, bone accretion in youth with type 2 diabetes may fail to keep pace with that of their peers of similar weight and body composition. LBMI is a known positive predictor of BMC and aBMD outcomes, but both the type 2 diabetes and obese groups had significantly lower aBMD $z$ scores for a given LBMI $z$ score compared with healthy weight control patients. Moreover, visceral fat was negatively associated with bone mass and density, highlighting a potential adverse influence of pathogenic fat depots on bone development.

Type 2 diabetes has historically been considered an adult-onset condition, and 
A

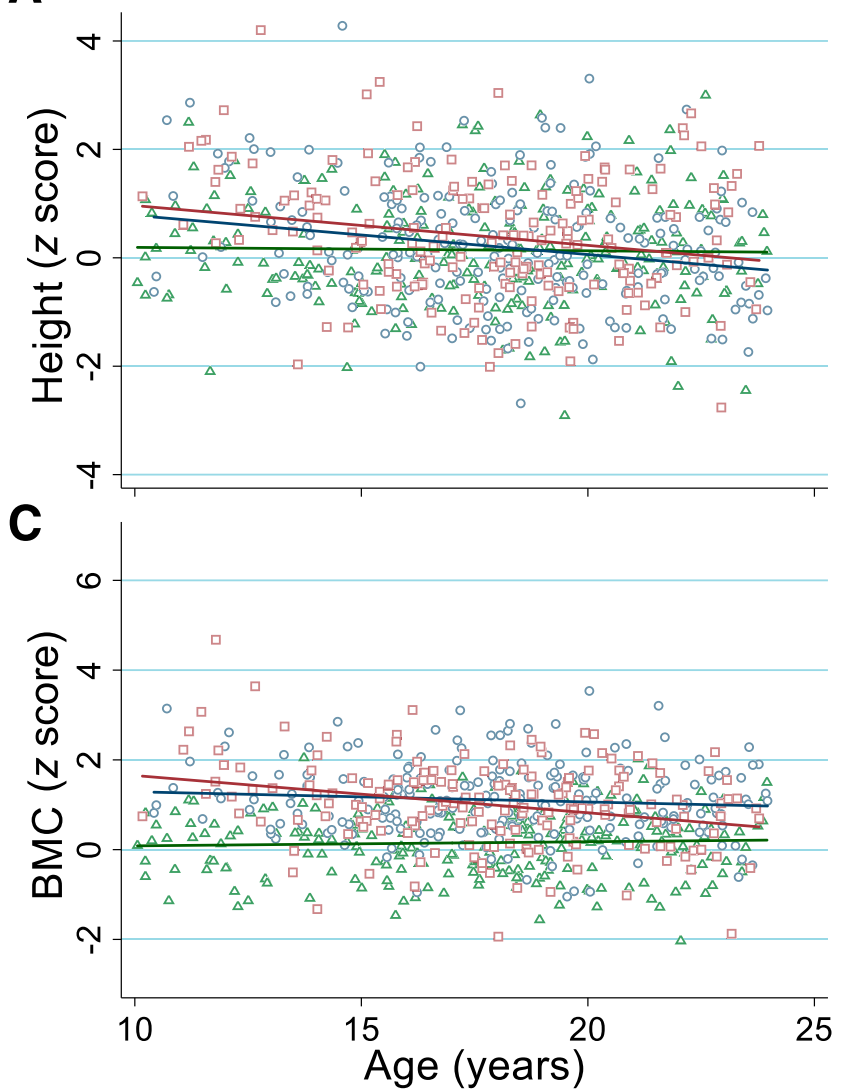

B

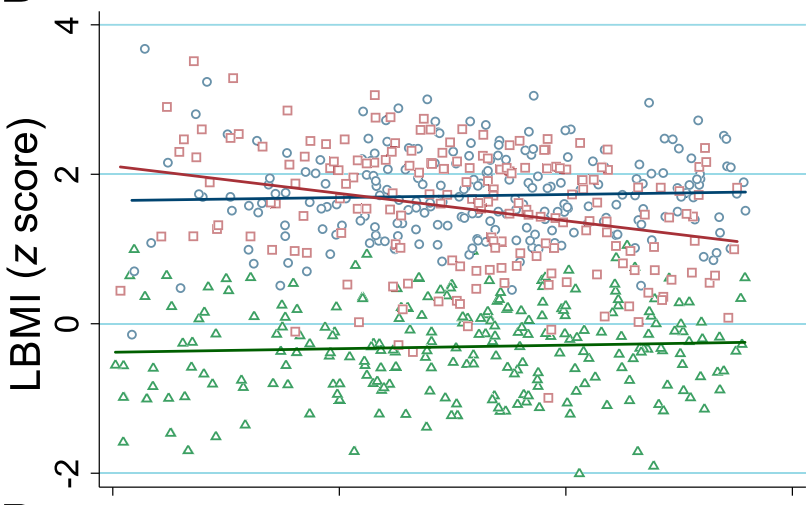

D

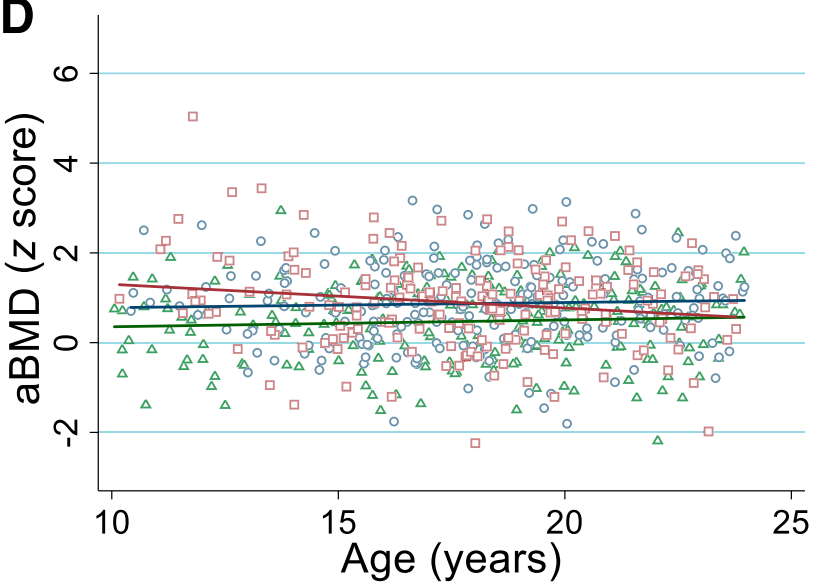

$\triangle$ Healthy Weight $\_$Obese $\square$ Type 2 Diabetes

Figure 1-Age-dependent differences in height $(A), \operatorname{LBMI}(B), \mathrm{BMC}(C)$, and aBMD $(D) z$ scores in youth with healthy weight (green triangles and green line), obesity (blue circles and blue line), and type 2 diabetes (red squares and red line). Regression lines are based on predicted values and are displayed for each group. LBMI is calculated as lean body mass ( $\mathrm{kg}$; assessed via DXA) divided by height $(\mathrm{cm})$ squared.

studies focusing on bone outcomes have been confined to adults. The lack of pediatric studies is concerning due to the increasing incidence of youth-onset type 2 diabetes, the accelerated rate of disease progression and development of comorbidities, and the use of therapies such as exogenous insulin that have been linked to increased fracture risk in adults (26). Importantly, childhood is a critical period of bone accrual, with peak bone mass being achieved around the age of 20 years $(2,3,17)$. In adults, individuals with type 2 diabetes had comparable BMD to control patients without diabetes with a similar BMI $(6,7,27)$. The simple between-group comparisons in our study generally agree with these adult observations since average bone $z$ scores were similar between those with type 2 diabetes and obesity. The $z$ scores account for expected age trends associated with growth and maturation. Unless perturbed by adverse health or environmental circumstances, $z$ score values should be stable across childhood, as demonstrated in our healthy weight and obese groups. The decline in bone $z$ scores relative to age in the type 2 diabetes group suggests a potentially adverse influence of type 2 diabetes on bone health irrespective of obesity. For example, at 10 and 24 years of age, predicted aBMD $z$ scores in the obese group were 0.8 and 0.9 , respectively, but in the type 2 diabetes group, predicted $\mathrm{aBMD} z$ scores were 1.3 and 0.6 , respectively. This finding is particularly concerning as it suggests that type 2 diabetes may compromise peak bone mass attainment (17). This was a cross-sectional study, so it is unclear whether bone $z$ scores indeed decrease throughout childhood and adolescence in youth with type 2 diabetes. Prospective studies are needed to further delineate the role of obesity and type 2 diabetes on peak bone mass acquisition during critical periods of growth.

Our observed age-related trends in bone $z$ scores are unlikely attributable to bone loss since this is a relatively rare phenomenon in childhood. Rather, this may be due to lesser gains in bone accretion, which was observed in a mouse model of early onset type 2 diabetes (14). Sexual maturation and stature likely also contributed, at least in part, to these differences. Obese and type 2 diabetes groups were taller than the healthy weight individuals until the later teenage years, consistent with earlier puberty and peak height velocity associated with obesity $(28,29)$. For these reasons, heightadjusted bone $z$ scores are reported in this study. Nevertheless, considering the close link between stature and bone mass during childhood, earlier maturation and taller stature could contribute to the increased bone mass in type 2 diabetes and obesity at younger versus older ages when cessation of rapid longitudinal growth in normally maturing youth is achieved (30).

Youth-onset type 2 diabetes is characterized by rapid declines in pancreatic $\beta$-cell function and insulin resistance (3). 

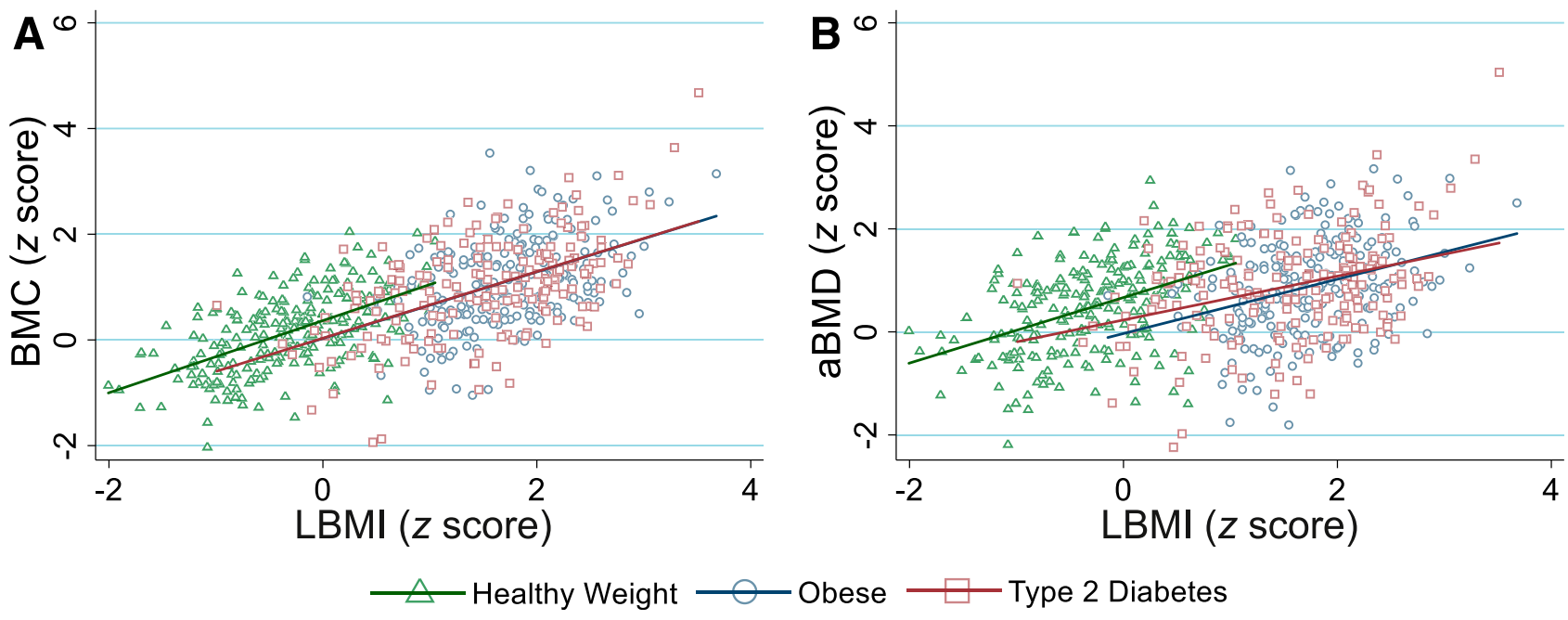

Figure 2-BMC $(A)$ and aBMD $(B)$ z scores relative to LBMI $z$ score in youth with healthy weight (green triangles and green line), obesity (blue circles and blue line), and type 2 diabetes (red squares and red line). Regression lines are based on predicted values and are displayed for each group. LBMI is calculated as lean body mass ( $\mathrm{kg}$; assessed via DXA) divided by height $(\mathrm{cm})$ squared.

Since insulin is a bone-anabolic hormone (31), manifestations in insulin secretion and insulin action could contribute to type 2 diabetes-related alterations in bone metabolism. Lower concentrations of bone formation markers have been reported in children with prediabetes (12) and in a murine model of early onset type 2 diabetes (14). Perhaps the age-related differences involve the duration of type 2 diabetes (Supplementary Fig. 1) and, thus, longer exposure to diabetes-related disturbances, nutritional inadequacies, physical inactivity, and pharmacologic or behavioral interventions and their effects on bone and body composition. Other cross-sectional studies have reported that the duration of type 2 diabetes might contribute to bone health deficits in adults (16), but this hypothesis necessitates rigorous longitudinal tracking in youth. In adults, disease-specific risk factors for fracture include diabetes duration $>5$ years, medications (e.g., insulin and glitazones), $\mathrm{HbA}_{1 \mathrm{c}}>7 \%$, and microvascular complications (e.g., neuropathy, retinopathy, and nephropathy) (32). Given these adult risk factors, youth with type 2 diabetes may be particularly vulnerable to skeletal deficits and fracture due to the more rapid onset of diabetes-related complications in youth compared with adulthood (33).

Bone tissue is responsive to loading resulting from excess body weight and skeletal muscle. On average, LBMI, a measure of skeletal muscle, was greater in the type 2 diabetes and obese groups versus the healthy weight group, and it was strongly and positively associated with bone $z$ scores. However, we observed a unique age-related trend in LBMI $z$ score in the type 2 diabetes group, which mirrors the bone mass and density findings discussed above. To illustrate, the predicted LBMI $z$ score was about 0.45 SDs greater among children in the type 2 diabetes group versus the obese group but about 0.66 SDs lower in adolescents and young adults in the type 2 diabetes group versus the obese group. Moreover, there was evidence of a potentially compromised muscle-bone relationship in youth with obesity and type 2 diabetes. On average, aBMD for a given LBMI $z$ score was about 0.5 SDs lower in the obese and type 2 diabetes groups versus the healthy weight control patients. This may reflect the inability of bone accrual to meet the needs to support greater body size and mechanical strain in the setting of excess adiposity. Consequently, these individuals might not have sufficient BMD and strength to withstand the excess loads that accompany a fall, thereby being vulnerable to fracture.

Abdominal visceral fat has been implicated in type 2 diabetes (34) and has been hypothesized to adversely influence BMD and geometry in childhood $(11,13)$. In our study, visceral fat was greater in the type 2 diabetes group versus the healthy weight and obese groups, and it was negatively associated with $\mathrm{BMC}$ and $\mathrm{aBMD}$. In contrast, subcutaneous fat was positively associated with BMC. Others have reported similar results involving the relationships between abdominal visceral fat versus subcutaneous fat with bone outcomes $(11,13,35)$. For instance, in otherwise healthy females ages $15-25$ years (mean BMI $24.2 \pm 4.3 \mathrm{~kg} / \mathrm{m}^{2}$ ), Gilsanz et al. (35) reported negative and positive relationships between abdominal visceral and subcutaneous fat, respectively, with femoral bone geometry and estimated bone strength. Interestingly, neither visceral nor subcutaneous fat were associated with bone outcomes in those with healthy weight in the current study, potentially due to the small variability in abdominal fat. Further, the divergent relationships between specific abdominal fat stores and bone likely reflect differences in metabolic activity between visceral and subcutaneous fat $(34,36)$. Insulin resistance has been identified as an intermediary in the link between increased abdominal fat and lower BMD in children (37), but the role of specific fat depots in bone metabolism requires further attention.

Diet and physical activity are key contributors to obesity and type 2 diabetes, and they play an important role in peak bone mass attainment (17). We observed differences in bone-related nutrients and physical activity that are suggestive of a potential contribution of these factors in our reported findings. Calcium is the main mineral constituent of skeletal tissue, and youth with obesity and type 2 diabetes had lower self-reported calcium intakes compared with healthy weight 
Table 2-Relationships of abdominal visceral and subcutaneous fat with bone $z$ scores in the obese and type 2 diabetes groups combined

\begin{tabular}{lccc} 
& $\beta$ & $P$ value & $R^{2}$ \\
\hline BMC (z score) & & & 0.11 \\
African American ancestry & -0.052 & 0.336 & \\
Age (years) & -0.187 & $<0.001$ & \\
Female sex & 0.137 & 0.008 & \\
Subcutaneous fat $\left(\mathrm{cm}^{2}\right)$ & 0.227 & $<0.001$ & \\
Visceral fat $\left(\mathrm{cm}^{2}\right)$ & -0.121 & 0.047 & 0.05 \\
aBMD $(z$ score) & & & \\
African American ancestry & -0.118 & 0.034 & \\
Age (years) & -0.048 & 0.329 & \\
Female sex & 0.163 & 0.002 & \\
Subcutaneous fat $\left(\mathrm{cm}^{2}\right)$ & 0.092 & 0.140 & \\
Visceral fat $\left(\mathrm{cm}^{2}\right)$ & -0.138 & 0.028 & \\
\hline
\end{tabular}

$\beta$, standardized regression coefficient.

peers. Vitamin D plays an active role in intestinal calcium absorption (38), and as reported in our study, individuals with excess adiposity are at increased risk for vitamin $D$ deficiency (39). After accounting for $25(\mathrm{OH}) \mathrm{D}$, the negative age-related trend in aBMD $z$ scores in youth with type 2 diabetes was attenuated, and increased visceral fat was no longer associated with lower aBMD $z$ scores, suggesting that dietary factors including calcium, vitamin $D$, and others might contribute to these relationships. Moreover, dynamic loading resulting from high-impact physical activity and muscle contraction promote bone mineralization and bone strength improvements, specifically in the setting of appropriate calcium intake (40). An earlier study from this cohort reported lower physical activity levels among the obese and type 2 diabetes groups compared with the healthy weight peers (41). Accordingly, poor diet and physical inactivity might independently and synergistically influence bone health in youth with obesity and related health conditions. Since the direct contributions of diet and physical activity on aBMD were beyond the scope of the current study, additional work is required to understand these relationships.

The large sample size and inclusion of both healthy weight and obese groups with normal glucose control were key strengths of this study. The betweengroup comparisons that did not account for age were suggestive of similar bone mass and density between the type 2 diabetes and obese groups. However, we identified significant age interactions that implicate type 2 diabetes as a potential detriment to bone density around the age of peak bone mass. Subsequent analyses showed that vitamin D status contributed to these age-related trends, but it is possible that other unidentified confounders were also involved in these relationships. Nevertheless, our results underscore the importance of testing for age and maturation effects in future studies to fully understand the effects of type 2 diabetes on the developing skeleton. There are currently no clinical guidelines for bone assessment specific to youth with obesity or type 2 diabetes. Regardless of clinical population, it is important to follow the pediatric positions set forth by the International Society for Clinical Densitometry, which recommend analyzing the whole-body (less head) DXA scans for bone health assessment in children and adolescents (42). Height-adjustment of bone $z$ scores is also recommended to account for stature-related confounding and helps account for maturation, supporting the use of this approach in children with taller stature and earlier maturation, such as those with obesity (43).

Although the whole-body region is recommended for pediatric DXA exams (44), BMD from DXA might not be sensitive to the myriad effects of diabetes on bone (27), thereby limiting our ability to observe differences between youth with obesity compared with those with type 2 diabetes. The majority of the human skeleton is comprised of cortical bone, so it is unknown how youth-onset type 2 diabetes relates to $\mathrm{BMC}$ and $\mathrm{aBMD}$ at skeletal sites that are rich in trabecular bone (e.g., the lumbar spine). Moreover, DXA is unable to assess bone microarchitecture, which entails characteristics of bone strength that are suspected to contribute to fracture etiology in adult type 2 diabetes (45). Yu et al. (27) reported inferior bone microarchitecture in African American women with type 2 diabetes versus control patients without diabetes despite having similar bone $B M D$ via DXA. These findings highlight the importance of incorporating measures of bone microarchitecture into future studies to maximize statistical power and to understand the cumulative influence of type 2 diabetes on the growing skeleton. The cross-sectional study design was also a limitation of this study, as we cannot draw causal conclusions from our results. Additionally, because of the potential bias associated with self-reported maturation stage, these results should be interpreted with caution (46). Nevertheless, the taller stature in children with obesity and type 2 diabetes that was attenuated in adolescents is consistent with obesity-associated earlier maturation and peak height velocity $(28,29)$. Moreover, youth-onset type 2 diabetes is associated with excess adiposity, but it is not exclusive to individuals with obesity. About $85 \%$ of U.S. youth with type 2 diabetes were reported to have obesity (47) compared with about $89 \%$ in the current study. Since this variability in BMI can complicate the interpretation of our findings, we performed sensitivity analyses excluding individuals with type 2 diabetes who were not obese. Overall, these results did not appreciably differ from those of our main analyses. Finally, data on socioeconomic status were not available, which limits the generalizability of our findings.

In conclusion, results from this study suggest that type 2 diabetes in youth may have a detrimental effect on bone accrual during the critical window of peak bone mass attainment irrespective of obesity status. Despite skeletal muscle being a key determinant of childhood bone mass, we observed a disconnect in the musclebone relationship in the obese and type 2 diabetes groups. Visceral fat has been implicated in type 2 diabetes progression, and those with increased abdominal visceral fat tended to have lower BMD. Prospective studies are needed to further understand the effects of type 2 diabetes on bone accrual during critical periods of growth as these discrepancies may have lasting effects on risk of fragility fractures later in life. Additionally, it is critical that subsequent studies evaluate characteristics of bone microarchitecture and strength since fracture etiology in type 2 
diabetes likely involves skeletal features that cannot be assessed through standard clinical methods (45).

Funding. J.M.K. is funded through the National Center for Advancing Translational Sciences of the National Institutes of Health (TL1TR001880), the American Diabetes Association (1-19-PDF-129), and the Endocrine Fellows Foundation. The original study was funded by the National Institutes of Health National Heart, Lung, and Blood Institute (R01HL076269).

Duality of Interest. No potential conflicts of interest relevant to this article were reported. Author Contributions. J.M.K. wrote the manuscript and performed statistical analyses. J.M.K., E.M.U., and B.S.Z. developed the study objectives. All authors contributed to interpretation of results, reviewed and edited the manuscript, approved the final version of the manuscript, and agreed to the submission and publication of the manuscript. J.M.K. is the guarantor of this work and, as such, had full access to all the data in the study and takes responsibility for the integrity of the data and the accuracy of the data analysis. Prior Presentation. Parts of this study were presented at the Children's Hospital of Philadelphia Research Poster Day, Philadelphia, PA, 21 May 2019; the Endocrine Fellows Foundation Fellows Forum, Orlando, FL, 18-19 September 2019; the American Society for Bone and Mineral Research Annual Meeting, Orlando, FL, 20-23 September 2019; and the Endocrine Fellows Foundation Research Meeting, Southlake, TX, 6-8 February 2020.

\section{References}

1. Pinhas-Hamiel O, Zeitler P. The global spread of type 2 diabetes mellitus in children and adolescents. J Pediatr 2005;146:693-700

2. Mayer-Davis EJ, Lawrence JM, Dabelea D, et al.; SEARCH for Diabetes in Youth Study. Incidence trends of type 1 and type 2 diabetes among youths, 2002-2012. N Engl J Med 2017;376:1419-1429 3. Hannon TS, Arslanian SA. The changing face of diabetes in youth: lessons learned from studies of type 2 diabetes. Ann NY Acad Sci 2015;1353:113-137 4. Urbina EM, Kimball TR, McCoy CE, Khoury PR, Daniels SR, Dolan LM. Youth with obesity and obesity-related type 2 diabetes mellitus demonstrate abnormalities in carotid structure and function. Circulation 2009;119:2913-2919

5. Copeland KC, Zeitler P, Geffner M, et al.; TODAY Study Group. Characteristics of adolescents and youth with recent-onset type 2 diabetes: the TODAY cohort at baseline. J Clin Endocrinol Metab 2011;96:159-167

6. Schwartz AV, Vittinghoff $E$, Bauer DC, et al.; Study of Osteoporotic Fractures (SOF) Research Group; Osteoporotic Fractures in Men (MrOS) Research Group; Health, Aging, and Body Composition (Health $A B C$ ) Research Group. Association of BMD and FRAX score with risk of fracture in older adults with type 2 diabetes. JAMA 2011; 305:2184-2192

7. Leslie WD, Rubin MR, Schwartz AV, Kanis JA. Type 2 diabetes and bone. J Bone Miner Res 2012;27:2231-2237

8. Pollock NK. Childhood obesity, bone development, and cardiometabolic risk factors. Mol Cell Endocrinol 2015;410:52-63
9. Kindler JM, Pollock NK, Laing EM, et al. Insulin resistance and the IGF-I-cortical bone relationship in children ages 9 to 13 years. J Bone Miner Res 2017;32:1537-1545

10. Afghani A, Cruz ML, Goran Ml. Impaired glucose tolerance and bone mineral content in overweight latino children with a family history of type 2 diabetes. Diabetes Care 2005;28:372378

11. Pollock NK, Bernard PJ, Gutin B, Davis CL, Zhu $\mathrm{H}$, Dong Y. Adolescent obesity, bone mass, and cardiometabolic risk factors. J Pediatr 2011;158: 727-734

12. Pollock NK, Bernard PJ, Wenger $K$, et al. Lower bone mass in prepubertal overweight children with prediabetes. J Bone Miner Res 2010;25:2760-2769

13. Glass NA, Torner JC, Letuchy EM, et al. Does visceral or subcutaneous fat influence peripheral cortical bone strength during adolescence? A longitudinal study. J Bone Miner Res 2018;33: 580-588

14. Devlin MJ, Van Vliet M, Motyl K, et al. Earlyonset type 2 diabetes impairs skeletal acquisition in the male TALLYHO/JngJ mouse. Endocrinology 2014:155:3806-3816

15. Rudman HA, Birrell F, Pearce MS, et al. Obesity, bone density relative to body weight and prevalent vertebral fracture at age 62 years: the Newcastle thousand families study. Osteoporos Int 2019;30:829-836

16. Starr JF, Bandeira LC, Agarwal S, et al. Robust trabecular microstructure in type 2 diabetes revealed by individual trabecula segmentation analysis of HR-pQCT images. J Bone Miner Res 2018;33:1665-1675

17. Weaver $\mathrm{CM}$, Gordon $\mathrm{CM}$, Janz KF, et al. The National Osteoporosis Foundation's position statement on peak bone mass development and lifestyle factors: a systematic review and implementation recommendations [published correction appears in Osteoporos Int 2016;27:1387]. Osteoporos Int 2016;27:1281-1386

18. Gao Z, Khoury PR, McCoy CE, et al. Adiposity has no direct effect on carotid intima-media thickness in adolescents and young adults: use of structural equation modeling to elucidate indirect \& direct pathways. Atherosclerosis 2016; 246:29-35

19. American Diabetes Association. Diagnosis and classification of diabetes mellitus. Diabetes Care 2014;37(Suppl. 1):S81-S90

20. Kuczmarski RJ, Ogden $\mathrm{CL}$, Grummer-Strawn LM, et al. CDC growth charts: United States. Adv Data 2000;314:1-27

21. Tanner J. Growth and Adolescence. 2nd ed. Oxford, U.K., Blackwell Scientific Publications, 1962

22. Jha $P$, Dolan LM, Khoury PR, Urbina EM, Kimball TR, Shah AS. Low serum vitamin D levels are associated with increased arterial stiffness in youth with type 2 diabetes. Diabetes Care 2015; 38:1551-1557

23. Zemel BS, Kalkwarf HJ, Gilsanz V, et al. Revised reference curves for bone mineral content and areal bone mineral density according to age and sex for black and non-black children: results of the bone mineral density in childhood study. J Clin Endocrinol Metab 2011;96:3160-3169

24. Weber DR, Moore RH, Leonard MB, Zemel $B S$. Fat and lean BMI reference curves in children and adolescents and their utility in identifying excess adiposity compared with BMI and percentage body fat. Am J Clin Nutr 2013;98:49-56 25. Zemel BS, Leonard MB, Kelly A, et al. Height adjustment in assessing dual energy $\mathbf{x}$-ray absorptiometry measurements of bone mass and density in children. J Clin Endocrinol Metab 2010; 95:1265-1273

26. Melton LJ III, Leibson CL, Achenbach SJ, Therneau TM, Khosla S. Fracture risk in type 2 diabetes: update of a population-based study. J Bone Miner Res 2008;23:1334-1342

27. Yu EW, Putman MS, Derrico N, AbrishamanianGarcia G, Finkelstein JS, Bouxsein ML. Defects in cortical microarchitecture among African-American women with type 2 diabetes. Osteoporos Int 2015; 26:673-679

28. Ohlsson C, Bygdell M, Celind J, et al. Secular trends in pubertal growth acceleration in Swedish boys born from 1947 to 1996 . JAMA Pediatr 2019;173:860-865

29. De Leonibus C, Marcovecchio ML, Chiavaroli V, de Giorgis T, Chiarelli F, Mohn A. Timing of puberty and physical growth in obese children: a longitudinal study in boys and girls. Pediatr Obes 2014;9:292-299

30. McCormack SE, Cousminer DL, Chesi A, et al. Association between linear growth and bone accrual in a diverse cohort of children and adolescents. JAMA Pediatr 2017;171:e171769

31. Pramojanee SN, Phimphilai M, Chattipakorn $\mathrm{N}$, Chattipakorn SC. Possible roles of insulin signaling in osteoblasts. Endocr Res 2014;39:144151

32. Ferrari SL, Abrahamsen B, Napoli N, et al.; Bone and Diabetes Working Group of IOF. Diagnosis and management of bone fragility in diabetes: an emerging challenge. Osteoporos Int 2018;29:2585-2596

33. Zeitler P. Progress in understanding youthonset type 2 diabetes in the United States: recent lessons from clinical trials. World J Pediatr 2019; 15:315-321

34. Tchernof A, Després JP. Pathophysiology of human visceral obesity: an update. Physiol Rev 2013;93:359-404

35. Gilsanz V, Chalfant J, Mo AO, Lee DC, Dorey FJ, Mittelman SD. Reciprocal relations of subcutaneous and visceral fat to bone structure and strength. J Clin Endocrinol Metab 2009;94:33873393

36. McLaughlin T, Lamendola C, Liu A, Abbasi F. Preferential fat deposition in subcutaneous versus visceral depots is associated with insulin sensitivity. J Clin Endocrinol Metab 2011;96: E1756-E1760

37. Kindler JM, Lobene AJ, Vogel KA, et al. Adiposity, insulin resistance, and bone mass in children and adolescents. J Clin Endocrinol Metab 2019;104:892-899

38. DeLuca HF, Sicinski RR, Tanaka Y, Stern PH, Smith CM. Biological activity of 1,25-dihydroxyvitamin D2 and 24-epi-1,25-dihydroxyvitamin D2. Am J Physiol 1988;254:E402-E406

39. Vranić L, Mikolašević I, Milić S. Vitamin D deficiency: consequence or cause of obesity? Medicina (Kaunas) 2019;55:541

40. Daly RM, Duckham RL, Gianoudis J. Evidence for an interaction between exercise and nutrition for improving bone and muscle health. Curr Osteoporos Rep 2014;12:219-226

41. Edwards NM, Daniels SR, Claytor RP, et al. Physical activity is independently associated with multiple 
measures of arterial stiffness in adolescents and young adults. Metabolism 2012;61:869-872 42. Crabtree NJ, Arabi A, Bachrach LK, et al.; International Society for Clinical Densitometry. Dual-energy X-ray absorptiometry interpretation and reporting in children and adolescents: the revised 2013 ISCD Pediatric Official Positions. J Clin Densitom 2014;17:225-242

43. Wren TA, Kalkwarf HJ, Zemel BS, et al.; Bone Mineral Density in Childhood Study Group. Longitudinal tracking of dual-energy $\mathrm{X}$-ray absorptiometry bone measures over 6 years in children and adolescents: persistence of low bone mass to maturity. J Pediatr 2014;164:1280-1285

44. Gordon CM, Leonard MB, Zemel BS; International Society for Clinical Densitometry. 2013 Pediatric Position Development Conference: executive summary and reflections [published correction appears in J Clin Densitom 2014;17: 517]. J Clin Densitom 2014;17:219-224

45. Patsch JM, Burghardt AJ, Yap SP, et al. Increased cortical porosity in type 2 diabetic postmenopausal women with fragility fractures. J Bone Miner Res 2013;28:313-324 46. Lee K, Valeria B, Kochman C, Lenders CM. Self-assessment of height, weight, and sexual maturation: validity in overweight children and adolescents. J Adolesc Health 2006;39:346-352 47. Kim G, Divers J, Fino NF, et al. Trends in prevalence of cardiovascular risk factors from 2002 to 2012 among youth early in the course of type 1 and type 2 diabetes. The SEARCH for Diabetes in Youth Study. Pediatr Diabetes 2019;20:693-701 